\title{
Multidimensional Coded Modulation in Block-Fading Channels
}

\author{
Albert Guillén i Fàbregas and Giuseppe Caire
}

\begin{abstract}
We study the problem of constructing coded modulation schemes over multidimensional signal sets in Nakagami- $m$ block-fading channels. In particular, we consider the optimal diversity reliability exponent of the error probability when the multidimensional constellation is obtained as the rotation of classical complex-plane signal constellations. We show that multidimensional rotations of full dimension achieve the optimal diversity reliability exponent, also achieved by Gaussian constellations. Multidimensional rotations of full dimension induce a large decoding complexity, and in some cases it might be beneficial to use multiple rotations of smaller dimension. We also study the diversity reliability exponent in this case, which yields the optimal rate-diversity-complexity tradeoff in block-fading channels with discrete inputs.
\end{abstract}

A. Guillén i Fàbregas is with the Signal Processing and Communications Laboratory, Department of Engineering, University of Cambridge, Trumpington Street, Cambridge CB2 1PZ, UK, e-mail: guillen@ieee.org.

G. Caire is with the Electrical Engineering Department, University of Southern California, 3740 McClintock Ave., Los Angeles, CA 90089, USA, e-mail: caire@usc.edu.

The work by A. Guillén i Fàbregas work has been supported in part by the Australian Research Council under ARC grants DP0558861 and RN0459498. 


\section{INTRODUCTION}

Rotated multidimensional constellations in fading channels were proposed in [1], [2] as a means of achieving high reliability with uncoded modulation in fading channels. Since, rotated constellations have been extensively studied, and have been shown to be an effective technique to achieve full-rate and full-diversity transmission in fading channels [3], [4], [5], [6]. Traditionally, rotated constellations have always been studied uncoded, with the exception of some recent works for the multiple-input multiple-output (MIMO) channel [7], [8].

In this work, we study the problem of constructing general coded modulation schemes over multidimensional signal sets, obtained by rotating classical complex-plane signal constellations, for block-fading channels with $B$ fading blocks (or degrees of freedom) per codeword [9]. The block-fading channel is a useful model for transmission over slowly varying fading channels, such as orthogonal frequency division multiplexing (OFDM) or slow time-frequency-hopped systems such as GSM or EDGE.

Despite the elegance of full-diversity rotations of dimension $B$, they induce large decoding complexity since the set of candidate points for detection at a given time instant is exponential with $B$. In fact, when uncoded rotations are used, the sphere decoder [10] is usually employed to avoid exhaustive search over all candidate points. However, when coded modulation is used, the code itself can help to achieve full diversity. This means that sometimes rotations of smaller dimension $N<B$ might be sufficient. Also in the coded case, soft information should be provided to the decoder and this further complicates the problem. As a matter of fact, despite the recent advances in soft-output sphere decoding techniques [11], most of the proposed techniques still show some limitations in some cases, which might be undesirable in practice. Therefore, in practice, one might be interested in using rotations of dimension smaller than $B$, in order to establish the tradeoff between diversity, rate, constellation size and complexity induced by the rotations.

In this correspondence, we study the reliability exponent, namely, the optimal exponent of the error probability of such schemes with the signal-to-noise ratio (SNR), and illustrate the ratediversity-complexity tradeoff for coded modulation schemes constructed over multidimensional signal sets. 


\section{System ModeL}

We consider a single-input single-output block-fading channel with $B$ fading blocks, and is defined as follows,

$$
\mathbf{y}_{b}=\sqrt{\mathrm{SNR}} h_{b} \mathbf{x}_{b}+\mathbf{z}_{b} \quad b=1, \ldots, B
$$

where $h_{b} \in \mathbb{C}$ is the $b$-th fading coefficient, $\mathbf{y}_{b} \in \mathbb{C}^{L}$ is the received signal vector corresponding to fading coefficient $b, \mathbf{x}_{b} \in \mathbb{C}^{L}$ is the portion of codeword allocated to block $b$ and $\mathbf{z}_{b} \in \mathbb{C}^{L}$ is the vector of i.i.d. noise samples $\sim \mathcal{N}_{\mathbb{C}}(0,1)$. We assume that the transmitted signal is normalized in energy, i.e., $\mathbb{E}\left[|x|^{2}\right]=1$. Hence, SNR is the average received SNR.

We assume that the fading coefficients are i.i.d. from block to block and from codeword to codeword, and that they are perfectly known at the receiver, i.e, perfect channel state information (CSI). Since the channel coefficients are perfectly known to the receiver, we assume that the phase of the fading has been corrected. We also assume that the magnitudes of the channel coefficients follow a Nakagami- $m$ distribution

$$
p_{|h|}(\xi)=\frac{2 m^{m} \xi^{2 m-1}}{\Gamma(m)} e^{-m \xi^{2}}
$$

for $m>01$ where $\Gamma(\xi) \triangleq \int_{0}^{+\infty} t^{\xi-1} e^{-t} d t$ is the Gamma function [13]. By analizing Nakagami$m$ fading, we can recover the analysis for a large class of fading statistics, including Rayleigh fading by setting $m=1$ and Rician fading with parameter $K$ by setting $m=(K+1)^{2} /(2 K+1)$ [14]. We can express (1) in matrix form as

$$
\mathbf{Y}=\sqrt{\mathrm{SNR}} \mathbf{H} \mathbf{X}+\mathbf{Z}
$$

where $\mathbf{Y}=\left[\mathbf{y}_{1}, \ldots, \mathbf{y}_{B}\right]^{T}, \mathbf{X}=\left[\mathbf{x}_{1}, \ldots, \mathbf{x}_{B}\right]^{T}=\left[\mathbf{X}_{1}, \ldots, \mathbf{X}_{L}\right], \mathbf{Z}=\left[\mathbf{z}_{1}, \ldots, \mathbf{z}_{B}\right]^{T} \in \mathbb{C}^{B \times L}$ and $\mathbf{H}=\operatorname{diag}\left(h_{1}, \ldots, h_{B}\right) \in \mathbb{C}^{B \times B}$.

We consider that codewords $\mathrm{X}$ form a coded modulation scheme $\mathcal{X} \subset \mathbb{C}^{B \times L}$. In particular, we consider that $\mathcal{X}$ is obtained as the concatenation of a binary code $\mathcal{C} \in \mathbb{F}_{2}^{n}$ of rate $r$, a modulation over the signal constellation $\mathcal{S} \in \mathbb{C}$ with $M=\log _{2}|\mathcal{S}|$, and $K$ rotations $\mathbf{M}_{k} \in \mathbb{C}^{N \times N}$ with $K N=B$ (see Figure 1). In particular we have that at time $\ell=1, \ldots, L$

$$
\mathbf{x}_{\ell, k}=\mathbf{M}_{k} \mathbf{s}_{\ell, k}
$$

\footnotetext{
${ }^{1}$ The literature usually considers $m \geq 0.5$ [12]. However, the distribution is well defined and reliable communication is possible for $0<m<0.5$.
} 
where $\mathbf{s}_{\ell, k}=\left(s_{\ell, k, 1}, \ldots, s_{\ell, k, N}\right)^{T} \in \mathcal{S}^{N}$ is the vector of complex-plane signal constellation symbols that is rotated by the $k$-th rotation matrix, $\mathbf{x}_{\ell, k}=\left(x_{\ell, k, 1}, \ldots, x_{\ell, k, N}\right)^{T}$ is the portion of transmitted signal at time $\ell$ that has been rotated by the $k$-th rotation, and

$$
\mathbf{x}_{\ell}=\left[\mathbf{x}_{\ell, 1}^{T}, \ldots, \mathbf{x}_{\ell, K}^{T}\right]^{T}
$$

is the transmitted signal at time $\ell$. The rotation matrices are unitary, i.e., $\mathbf{M}_{k} \mathbf{M}_{k}^{\dagger}=\mathbf{I}$. We will be interested in full-fiversity rotations, namely, rotation matrices $\mathbf{M}$ for which $\forall \mathbf{s}, \mathbf{s}^{\prime} \in \mathcal{S}^{N}, \mathbf{s} \neq \mathbf{s}^{\prime}$

$$
\mathrm{M}\left(\mathrm{s}-\mathrm{s}^{\prime}\right) \neq \mathbf{0}
$$

componentwise. This implies that, if the vector $s-s^{\prime}$ has any number of non-zero components, its rotated version $\mathbf{M}\left(\mathbf{s}-\mathbf{s}^{\prime}\right)$ will have all non-zero components. The rate in bits per channel use of this scheme is $R=r M$. This general formulation includes the case where only one single rotation of dimension $B$ is used, as well as the other extreme, with $B$ trivial rotations of dimension $N=1$ (the non-rotated case).

Definition 1: The block-diversity of a coded modulation scheme $\mathcal{X} \subset \mathbb{C}^{B \times L}$ is defined as

$$
\delta=\min _{\substack{\mathbf{X}, \mathbf{X}^{\prime} \in \mathcal{X} \\ \mathbf{X}^{\prime} \neq \mathbf{X}}}\left|\left\{b \in(1, \ldots, B) \mid \mathbf{x}_{b} \neq \mathbf{x}_{b}^{\prime}\right\}\right| .
$$

In words, the block diversity is the minimum number of nonzero rows of $\mathbf{X}-\mathbf{X}^{\prime}$ for any pair of codewords $\mathrm{X}^{\prime} \neq \mathrm{X} \in \mathcal{X}$.

Proposition 1: Given a coded modulation scheme $\mathcal{X} \subset \mathbb{C}^{B \times L}$, the block diversity is upperbounded by

$$
\delta \leq N\left(1+\left\lfloor\frac{B}{N}\left(1-\frac{R}{M}\right)\right\rfloor\right) .
$$

Proof: The result follows from the straightforward application of the Singleton bound to the coded modulation $\mathcal{X}$ seen as a code of block-length $K$, over an alphabet of size $2^{M N L}$.

We will say that a code is blockwise maximum-distance separable (MDS) if it attains the Singleton bound of Proposition 11 with equality.

\section{Outage Probability}

Strictly speaking, the channel defined in (1) is not information stable and has zero capacity for any finite $B$ [15], since there is a non-zero probability that the transmitted message is detected 
in error. For sufficiently large $L$, the word error probability $P_{e}(\mathrm{SNR}, \mathcal{X})$ of any coding scheme $\mathcal{X} \subset \mathbb{C}^{B \times L}$ is lowerbounded by the information outage probability [9], [16], given by

$$
P_{e}(\mathrm{SNR}, \mathcal{X}) \geq P_{\text {out }}(\mathrm{SNR}, R) \triangleq \operatorname{Pr}(I(\mathrm{SNR}, \mathbf{H}) \leq R)
$$

where $I(\mathrm{SNR}, \mathbf{H})$ is the input-output mutual information of the channel for a given fading realization $\mathbf{H}$. In this work, we will study the behavior of $P_{\text {out }}(\mathrm{SNR}, R)$ for large SNR. In this regime, the optimal power allocation when no CSI is available at the transmitter, corresponds to evenly distributing the available power across all $B$ blocks. In the case of uniform allocation, and for a fixed $\mathbf{H}$, the outage probability is minimized when the entries of $\mathbf{X} \in \mathcal{X}$ are i.i.d. Gaussian $\sim \mathcal{N}_{\mathbb{C}}(0,1)$. In this case [17]

$$
I(\mathrm{SNR}, \mathbf{H})=\frac{1}{B} \sum_{b=1}^{B} \log _{2}\left(1+\operatorname{SNR} \gamma_{b}\right) .
$$

With the coded modulation scheme shown in Figure 1 is used (assuming uniform inputs), we can express the instantaneous mutual information in bits per channel use for a given channel realization $\mathbf{H}$ as

$$
I(\mathrm{SNR}, \mathbf{H})=\frac{1}{K} \sum_{k=1}^{K} \frac{1}{N} I_{k}\left(\mathrm{SNR}, \mathbf{H}_{k}\right)=\frac{1}{B} \sum_{k=1}^{K} I_{k}\left(\mathrm{SNR}, \mathbf{H}_{k}\right)
$$

where

$$
I_{k}\left(\mathrm{SNR}, \mathbf{H}_{k}\right)=M N-\frac{1}{2^{M N}} \sum_{\mathbf{s} \in \mathcal{S}^{N}} \mathbb{E}_{\mathbf{z}}\left[\log _{2}\left(1+\sum_{\mathbf{s}^{\prime} \neq \mathbf{s}} e^{-\left\|\sqrt{\mathrm{SNR}} \mathbf{H}_{k} \mathbf{M}_{k}\left(\mathbf{s}-\mathbf{s}^{\prime}\right)+\mathbf{z}\right\|^{2}+\|\mathbf{z}\|^{2}}\right)\right]
$$

denotes the mutual information of the $N \times N$ MIMO channel induced by the $k$-th rotation, and $\mathbf{H}_{k}=\operatorname{diag}\left(h_{(k-1) N+1}, \ldots, h_{k N}\right) \in \mathbb{C}^{N \times N}$ are the channel coefficients used by rotation $k$. Note that for small $N$, the expectation over the noise vector $\mathbf{z}$ in (9) can be efficiently computed using the Gauss-Hermite quadrature rules [13].

Note that concatenating a Gaussian random code with a rotation of dimension $B$ brings no benefit in terms of exponent nor mutual information. In fact, the output of the rotated Gaussian i.i.d. vector is also a Gaussian i.i.d. vector with identical distribution, provided that the rotation matrix is unitary. Therefore, the mutual information

$$
I(\mathrm{SNR}, \mathbf{H})=\frac{1}{B} \log _{2} \operatorname{det}\left(\mathbf{I}+\operatorname{SNR} \mathbf{H M M}^{\dagger} \mathbf{H}^{\dagger}\right)=\frac{1}{B} \sum_{b=1}^{B} \log _{2}\left(1+\operatorname{SNR} \gamma_{b}\right) .
$$


is the same than without rotation, and so is therefore the corresponding diversity exponent. Rotations are usually seen as information lossless, when in fact they are simply not needed when combined with Gaussian inputs.

Figure 2 shows the mutual information with Gaussian inputs, unrotated 16-QAM (identity rotation) and rotated 2 16-QAM in a block-fading channel with $B=4$ blocks and $h_{1}=1.5$ and $h_{2}=h_{3}=h_{4}=0.1$. This choice of the channel coefficients is particularly interesting since 3 out of the 4 components are in a deep fade. Rotations of dimension $N$ yield vanishing (with SNR) error probability whenever there are up to $N-1$ deeply faded blocks. The mutual information achieved by the rotated 16-QAM is very close to that attained by the Gaussian distribution for a range of SNR significantly wider than unrotated the unrotated 16-QAM. For example, at $\mathrm{SNR}=25 \mathrm{~dB}$, the Krüskemper rotation gains 1 bit of information with respect to unrotated 16QAM. Combining 2 cyclotomic rotations of dimension $N=2$ brings also significant information gains with respect to unrotated 16-QAM. As we shall see, this effect brings substantial exponent benefits with respect to the unrotated case. We also appreciate some difference between optimal Krüskemper and the mixed $(2 \times 2)$ rotations, especially at low rates. As a matter of fact, rotations provide only mutual information advantages at high rates. At low rates, unrotated transmission performs almost as well with much less decoding complexity.

\section{Optimal ReLiability}

We define the diversity reliability exponent of a given coded modulation scheme $\mathcal{X}$ as

$$
d_{\mathcal{X}}=\lim _{\mathrm{SNR} \rightarrow+\infty}-\frac{\log P_{e}(\mathrm{SNR}, \mathcal{X})}{\log \mathrm{SNR}}
$$

and the optimal diversity reliability exponent is

$$
d^{\star} \triangleq \sup _{\mathcal{X}} d_{\mathcal{X}}=\sup _{\mathcal{X}} \lim _{\mathrm{SNR} \rightarrow+\infty}-\frac{\log P_{e}(\mathrm{SNR}, \mathcal{X})}{\log \mathrm{SNR}}
$$

When no particular structure is imposed on the coded modulation scheme $\mathcal{X}$, we have the following result.

\footnotetext{
${ }^{2}$ Rotation matrices are extracted from [18]. This reference reports rotation matrices using the row convention used in [19]. In this paper, we use a column convention for lattice generator matrices, and therefore, matrices from [18] are transposed.
} 
Lemma 1: The diversity reliability exponent $d_{\mathcal{X}}$ of any coded modulation scheme $\mathcal{X}$ subject to the power constraint $\frac{1}{B L} \mathbb{E}\left[\|\mathbf{X}\|^{2}\right] \leq 1$ is upperbounded by

$$
d_{\mathcal{X}} \leq d^{\star}=m B
$$

The optimal diversity reliability exponent can be achieved by random Gaussian codes of rate $R>0$ with entries $\sim \mathcal{N}_{\mathbb{C}}(0,1)$. The optimal exponent $d^{\star}$ can also be achieved by random coded modulation schemes $\mathcal{X}$ of rate $R$ consisting of a random coded modulation scheme over a discrete signal constellation $\mathcal{S}$ of size $|\mathcal{S}|=2^{M}$ concatenated with a full-diversity rotation of dimension $B$, whenever $0 \leq \frac{R}{M}<1$.

Proof: The converse is proved in [20], [21]. Furthermore, [20], [21] also show that the random Gaussian ensemble achieves the optimal exponent. What is left to prove is that the random coded modulation scheme over a single full-diversity rotation of dimension $B$ achieves the same exponent. This is proved in Appendix III, by letting $N=B$.

Note that we have added the achievability with random coded modulation ensemble over the $B$-dimensional rotated constellation to illustrate that a coding scheme with discrete inputs can also achieve the optimal exponent. This result which is based on a divide and conquer approach, should be rather intuitive: the rotation of dimension $B$ takes care of achieving full diversity while the coding gain is then left to the outer coded modulation scheme over $\mathcal{S}$. When no rotations are used, the optimal diversity reliability exponent is given by the Singleton bound [21]

$$
d^{\star}=m\left(1+\left\lfloor B\left(1-\frac{R}{M}\right)\right\rfloor\right) .
$$

As shown in Figure 3 the advantage of rotations is clear: they can achieve optimal diversity reliability exponent for all the range of rates. Instead, when no rotations are used, the largest rate such that optimal diversity reliability exponent is achieved is $R=\frac{M}{B}$.

As outlined in the Introduction, full-diversity rotations induce large decoding complexity, since the size of set of candidate points at a given time instant is $2^{M B}$. We are therefore interested in characterizing the optimal diversity reliability exponent when rotations of smaller size $N<B$ are employed. We have the following results

Proposition 2: The diversity reliability exponent for the coded modulation schemes based on $K$ rotations of dimension $N$, in a Nakagami- $m$ block-fading channel with $B=K N$ blocks is 
upperbounded by

$$
d_{\mathcal{X}} \leq m N\left(1+\left\lfloor\frac{B}{N}\left(1-\frac{R}{M}\right)\right\rfloor\right)
$$

Proposition 3: The diversity reliability exponent in a Nakagami- $m$ block-fading channel with $B=K N$ of random coded modulation schemes based on $K$ rotations of dimension $N$ of length $L$ satisfying $\lim _{\mathrm{SNR} \rightarrow \infty} \frac{L(\mathrm{SNR})}{\mathrm{SNR}}=\lambda$, is lowerbounded by

$$
d_{\mathcal{X}} \geq \begin{cases}\lambda B M \log 2\left(1-\frac{R}{M}\right) & \text { if } 0 \leq \lambda N M \log 2<m \\ \min \left\{m N\left\lceil\frac{B}{N}\left(1-\frac{R}{M}\right)\right\rceil, m N\left\lfloor\frac{B}{N}\left(1-\frac{R}{M}\right)\right\rfloor\right. & \\ \left.+\lambda M \log 2\left(B\left(1-\frac{R}{M}\right)-N\left\lfloor\frac{B}{N}\left(1-\frac{R}{M}\right)\right\rfloor\right)\right\} & \text { otherwise. }\end{cases}
$$

Theorem 1: The optimal diversity reliability exponent for the coded modulation schemes based on $K$ rotations of dimension $N$, in a Nakagami- $m$ block-fading channel with $B=K N$ blocks is given by

$$
d_{\mathcal{X}}^{\star}=m N\left(1+\left\lfloor\frac{B}{N}\left(1-\frac{R}{M}\right)\right\rfloor\right)
$$

whenever $\frac{B}{N}\left(1-\frac{R}{M}\right)$ is not an integer.

Proof: Proposition 2 shows that

$$
d_{\mathcal{X}} \leq m N\left(1+\left\lfloor\frac{B}{N}\left(1-\frac{R}{M}\right)\right\rfloor\right)
$$

Letting $\lambda \rightarrow \infty$ in Proposition 3 shows that

$$
d_{\mathcal{X}} \geq m N\left\lceil\frac{B}{N}\left(1-\frac{R}{M}\right)\right\rceil .
$$

Noting that $\lceil x\rceil=\lfloor x\rfloor+1$ whenever $x$ is not an integer leads the desired result.

As we observe, Theorem 1 gives a dual result to that of [21] and shows that the optimal exponent is given by $m$ times the Singleton bound of (6), proving its optimality and separating the the roles of the channel distribution (through $m$ ) and of the code construction. The optimal codes are blockwise MDS in a channel with $B$ blocks. For $N>1$, Theorem 1 suggests that the optimal coding scheme is to use a coded modulation scheme constructed over $\mathcal{S}$ which is MDS in a block-fading channel with $K=\frac{B}{N}$ blocks concatenated with rotations of dimension $N$. In this case the MDS constraint on the code is relaxed, since it has to be MDS for a smaller number of blocks, at an expense of a decoding complexity increase. Theorem 1 implicitly introduces 
an equivalent channel model, namely, a block-fading channel with $K=\frac{B}{N}$, where each block has diversity $m N$. When $K=1, N=B$, there is only one single rotation of full dimension, Theorem 1 generalizes Lemma 1. The optimal coding scheme here does not need to be MDS. Therefore, Theorem 1 generalizes and proves the optimality of the modified Singleton bound introduced in [7].

Figure 4 shows the reliability exponents in the case of $B=8, m=0.5$ and $N=1,2,4$. The figure confirms the intuition behind such designs that the rotations should increase the reliability exponent. For example, for $\frac{R}{M}=\frac{1}{2}$, we have that with classical complex-plane inputs the reliability exponent is $d_{\mathcal{X}}^{\star}=m 5$, while for rotations with $N=2$ the exponent is $d_{\mathcal{X}}^{\star}=m 6$ and for $N=m 4$ the exponent is $d_{\mathcal{X}}^{\star}=m 8$, full diversity. This approach can be seen as a divideand-conquer approach, namely, the task of achieving diversity is split between both, the code $\mathcal{C}$ and the rotations. Figure 5 shows the diversity upper bound as well as the random coding lower bounds given in Propositions 2 and 3 , respectively. As we see, if $\lambda$ is increased, both bounds coincide in a larger support. Eventually, for $\lambda \rightarrow \infty$ they coincide wherever they are continuous.

To illustrate the performance benefits of rotations, Figures 6 and 7 show $P_{\text {out }}(\mathrm{SNR}, R)$ as a function of $\frac{E_{\mathrm{b}}}{N_{0}}$ in a block-fading channel with $m=1$ and $B=4$ for $R=2$, with Gaussian inputs (solid), discrete inputs (dotted), rotated discrete inputs with two cyclotomic rotations with $N=2$ (dash-dotted) and rotated discrete inputs with one Krüskemper rotation with $N=4$ (dashed). Gaussian inputs achieve the optimal exponent, namely $d^{\star}=B=4$, while unrotated inputs have $d_{\mathcal{X}}^{\star}=3$ [20]. As we observe from the curves, using two rotations of dimension $N=2$, not only allows to recover the largest possible exponent (in agreement with Theorem 1) but also brings a large gain in terms of gain. Using a rotation of dimension $N=4$ incurs much larger complexity and does not bring any exponent or gain improvements.

To illustrate that the above theoretical results are approachable with practical coding schemes, Figure 8 shows the error probability of rotated and unrotated systems with QPSK modulation using the $(5,7)_{8}$ convolutional code with 128 information bits per frame. The outage probabilities with Gaussian inputs (thick solid line), rotated QPSK inputs with one Krüskemper rotation of dimension $N=4$ (dashed line), rotated QPSK inputs with two cyclotomic rotations of dimension $N=2$ (dash-dotted) are shown for reference, as well as the performance of the unrotated scheme, whose corresponding outage probability has been removed for the sake of clarity. In the case of two rotations of dimension $N=2$, we separately use bit-interleaved coded modulation (BICM) 
[22] followed by a rotation on the outputs generated by generator polynoimial $5_{8}$ and $7_{8}$. Since the $(5,7)_{8}$ convolutional code has full-diversity in a block-fading channel with $K=2$ blocks, this blockwise operation allows the overall coding scheme to achieve full-diversity. A similar construction can be obtained using blockwise concatenated codes [20] or multiplexed turbocodes [23]. These coded modulation schemes will closely approach the outage probability of the channel for any (sufficiently large) block length. Rotated systems use exhaustive iterative decoders. Again, as we observe, the gain obtained by using rotations is significant. As a matter of fact, all systems using rotations show a steeper slope to that of the unrotated case. Furthermore, we observe that using a rotation of full dimension $N=4$ yields once more a small gain with respect to using two rotations of dimension $N=2$, while significantly increasing the decoding complexity. We also observe that, set-partitioning labeling yields some performance advantage over Gray labeling. From results not shown here, both Gray and set-partitioning show improved performance with the iterations. This is due to the the fact that rotations induce an equivalent MIMO channel, and the iterative decoder assists in iteratively removing the self-interference introduced by the rotation.

\section{CONCLUSIONS}

We have studied coded modulation schemes over Nakagami- $m$ block-fading channels with discrete input signal constellations. In particular, we have derived the optimal diversity reliability exponent for multidimensional signal constellations obtained from the rotation of classical complex-plane constellations, and we have shown that there is a tradeoff between the transmission rate, optimal achievable diversity, dimension of the rotations and size of the complex-plane signal constellation given by a modified form of the Singleton bound. Since using rotated constellations induces an increase in decoding complexity, the Singleton bound establishes the optimal ratediversiy-complexity tradeoff. We have shown that practical coding schemes can achieve the optimal rate-diversity-complexity tradeoff. 


\section{APPENDIX I}

\section{NOTATION}

In this appendix we introduce the main notation that will be used throughout the proofs of the various results. We will also state without proof some of the basic results that are needed for our proofs. The exponential equality $\doteq$ and inequalities $\dot{\geq}$ and $\dot{\leq}$ were introduced in [24]. We write

$$
f(z) \doteq z^{d}
$$

to indicate that

$$
\lim _{z \rightarrow \infty} \frac{\log f(z)}{\log z}=d .
$$

The exponential inequalities $\dot{\geq}$ and $\dot{\leq}$ are defined similarly. For vectors $\mathbf{x}, \mathbf{y} \in \mathbb{R}^{n}$, the notation $\mathbf{x} \prec \mathbf{y}$ is used to denote componentwise vector inequality, namely $x_{i}<y_{i}, i=1, \ldots, n$. The inequalities $\succ, \preceq, \succeq$ are used similarly. The function $\mathbb{1}\{\mathcal{E}\}$ is the indicator function of the event $\mathcal{E}$, namely, it $\mathbb{1}\{\mathcal{E}\}=1$ when the event $\mathcal{E}$ is true, and zero otherwise. Similarly to [24] we have the following.

Definition 2: The normalized fading coefficients are defined as

Then, from [21] we have that

$$
\alpha_{b} \triangleq-\frac{\log \gamma_{b}}{\log \mathrm{SNR}} \quad b=1, \ldots, B
$$

Proposition 4: The joint distribution of the vector $\boldsymbol{\alpha}=\left(\alpha_{1}, \ldots, \alpha_{B}\right)$ is given by

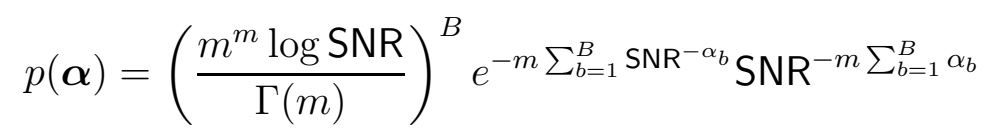

and in the limit for large SNR, behaves as

$$
p(\boldsymbol{\alpha}) \doteq \mathrm{SNR}^{-m \sum_{b=1}^{B} \alpha_{b}}
$$

for $\boldsymbol{\alpha} \in \mathbb{R}_{+}^{B}$. 


\section{APPENDIX II}

\section{ProOf of Proposition 2}

An upper bound to the mutual information yields a lower bound on the outage probability, and thus, an upper bound to the reliability exponent. We have,

$$
\begin{aligned}
I(\mathrm{SNR}, \mathbf{H}) & \leq \frac{1}{K} \sum_{k=1}^{K} \frac{1}{N} \min \left\{N M, \log \operatorname{det}\left(\mathbf{I}+\operatorname{SNR} \mathbf{H}_{k} \mathbf{M}_{k} \mathbf{M}_{k}^{\dagger} \mathbf{H}_{k}^{\dagger}\right)\right\} \\
& =\frac{1}{K} \sum_{k=1}^{K} \min \left\{M, \frac{1}{N} \sum_{n=1}^{N} \log \left(1+\operatorname{SNR} \gamma_{N(k-1)+n}\right)\right\} .
\end{aligned}
$$

Now, we can express the outage probability as

$$
\begin{aligned}
P_{\text {out }}(\mathrm{SNR}, R) & =\operatorname{Pr}(I(\mathrm{SNR}, \mathbf{H})<R) \\
& \geq \operatorname{Pr}\left(\frac{1}{K} \sum_{k=1}^{K} \min \left\{M, \frac{1}{N} \sum_{n=1}^{N} \log \left(1+\operatorname{SNR} \gamma_{N(k-1)+n}\right)\right\}<R\right) \\
& \doteq \operatorname{Pr}\left(\frac{1}{K} \sum_{k=1}^{K} \min \left\{M, \frac{\log \mathrm{SNR}}{N} \sum_{n=1}^{N}\left[1-\alpha_{N(k-1)+n}\right]_{+}\right\}<R\right) \\
& \geq \int_{\mathcal{O}_{\epsilon} \cap \mathbb{R}_{+}^{B}} \mathrm{SNR}^{-m \sum_{b=1}^{B} \alpha_{b}} d \boldsymbol{\alpha}
\end{aligned}
$$

where $[x]_{+}=\max (0, x)$ denotes the positive part of $x \in \mathbb{R}$ and

$$
\mathcal{O}_{\epsilon}=\left\{\boldsymbol{\alpha} \in \mathbb{R}^{B}: \frac{1}{K} \sum_{k=1}^{K} \mathbb{1}\left\{\boldsymbol{\alpha}_{k} \succeq \mathbf{1}+\boldsymbol{\epsilon}\right\}>1-\frac{R}{M}\right\}
$$

denotes the large SNR outage event, and where $\mathbf{1}=(1, \ldots, 1)$ and $\boldsymbol{\epsilon}=(\epsilon, \ldots, \epsilon)$ both of dimension $N$. Using Varadhan's integral lemma [25], we obtain,

$$
\begin{aligned}
d_{\mathcal{X}} \leq d_{\text {out }} & =-\lim _{\mathrm{SNR} \rightarrow \infty} \frac{1}{\log \mathrm{SNR}} \log \left(\int_{\mathcal{O}_{\epsilon} \cap \mathbb{R}_{+}^{B}} \mathrm{SNR}^{-m \sum_{b=1}^{B} \alpha_{b}} d \boldsymbol{\alpha}\right) \\
& =-\lim _{\mathrm{SNR} \rightarrow \infty} \frac{1}{\log \mathrm{SNR}} \log \left(\int_{\mathcal{O}_{\epsilon} \cap \mathbb{R}_{+}^{B}} \log \mathrm{SNR} \exp \left(-m \sum_{b=1}^{B} \alpha_{b}\right) d \boldsymbol{\alpha}\right) \\
& =\inf _{\mathcal{O}_{\epsilon} \cap \mathbb{R}_{+}^{B}}\left\{m \sum_{b=1}^{B} \alpha_{b}\right\}
\end{aligned}
$$

It is not difficult to show that $d_{\text {out }}=m \kappa N$, where $\kappa$ is the unique integer such that

$$
\kappa<K\left(1-\frac{R}{M}\right) \leq \kappa+1
$$


Hence we get that

$$
d_{\mathcal{X}} \leq d_{\text {out }}=m N\left(1+\left\lfloor\frac{B}{N}\left(1-\frac{R}{M}\right)\right\rfloor\right)
$$

which is precisely the desired result. 


\section{APPENDIX III}

\section{PROOF OF PROPOSITION 3}

For any two codewords $\mathbf{X}(0), \mathbf{X}(1) \in \mathcal{X}$, we can write that the pairwise error probability

$$
\begin{aligned}
P(\mathbf{X}(0) \rightarrow \mathbf{X}(1) \mid \mathbf{H}) & \leq \exp \left(-\frac{\mathrm{SNR}}{4}\|\mathbf{H}(\mathbf{X}(0)-\mathbf{X}(1))\|^{2}\right) \\
& =\prod_{k=1}^{K} \exp \left(-\frac{\mathrm{SNR}}{4}\left\|\mathbf{H}_{k} \mathbf{M}_{k}\left(\mathbf{S}_{k}(0)-\mathbf{S}_{k}(1)\right)\right\|^{2}\right)
\end{aligned}
$$

where $\mathbf{H}=\operatorname{diag}\left(\mathbf{H}_{1}, \ldots, \mathbf{H}_{K}\right)$. Assuming that the entries of $\mathbf{S}_{k}(0)$ and $\mathbf{S}_{k}(1)$ are chosen i.i.d. with uniform distribution over $\mathcal{S}$, we have that the ensemble pairwise error probability can be expressed as

$$
\overline{P(\mathbf{X}(0) \rightarrow \mathbf{X}(1) \mid \mathbf{H})} \leq \prod_{k=1}^{K}\left[\frac{1}{2^{2 M N}} \sum_{\mathbf{s} \in \mathcal{S}^{N}} \sum_{\mathbf{s}^{\prime} \in \mathcal{S}^{N}} \exp \left(-\frac{\mathrm{SNR}}{4}\left\|\mathbf{H}_{k} \mathbf{M}_{k}\left(\mathbf{s}-\mathbf{s}^{\prime}\right)\right\|^{2}\right)\right]^{L}
$$

Summing over the $2^{L B R}-1$ codewords different from the 0 message we have that

$$
\begin{aligned}
\overline{P_{e}(\mathrm{SNR} \mid \mathbf{H})} & \leq 2^{L B R} \prod_{k=1}^{K}\left[\frac{1}{2^{2 M N}} \sum_{\mathbf{s} \in \mathcal{S}^{N}} \sum_{\mathbf{s}^{\prime} \in \mathcal{S}^{N}} \exp \left(-\frac{\mathrm{SNR}}{4}\left\|\mathbf{H}_{k} \mathbf{M}_{k}\left(\mathbf{s}-\mathbf{s}^{\prime}\right)\right\|^{2}\right)\right]^{L} \\
& =\exp (-B L M \log 2 E(\mathrm{SNR}, \boldsymbol{\alpha}))
\end{aligned}
$$

where the exponent $E(\mathrm{SNR}, \boldsymbol{\alpha})$ is given by

$$
E(\operatorname{SNR}, \boldsymbol{\alpha})=1-\frac{R}{M}-\frac{1}{B M} \sum_{k=1}^{K} \log _{2}\left(1+\frac{1}{2^{M N}} \sum_{\mathbf{s}^{\prime} \neq \mathbf{s}} e^{-\frac{1}{4} \sum_{n=1}^{N} \mathrm{SNR}^{1-\alpha_{N(k-1)+n}}\left|\tilde{x}_{k, n}\right|^{2}}\right)
$$

and $\tilde{\mathbf{x}}_{k}=\mathbf{M}_{k}\left(\mathbf{s}-\mathbf{s}^{\prime}\right)=\left(\tilde{x}_{k, 1}, \ldots, \tilde{x}_{k, N_{k}}\right)^{T}$ is the rotated difference vector. We now assume that the rotation matrices have full diversity. That implies that all the components of the rotated difference vector $\tilde{\mathbf{x}}_{k}$ are different from zero. Then, for full diversity rotations we have that

$$
\begin{aligned}
& \log _{2}\left(1+\frac{1}{2^{M N}} \sum_{\mathbf{s}^{\prime} \neq \mathbf{s}} e^{\left.-\frac{\left|\max \left\{\tilde{\mathbf{x}}_{k}\right\}\right|^{2}}{4} \sum_{n=1}^{N} \mathrm{SNR}^{1-\alpha_{N(k-1)+n}}\right)}\right. \\
& \leq \log _{2}\left(1+\frac{1}{2^{M N}} \sum_{\mathbf{s}^{\prime} \neq \mathbf{s}} e^{\left.-\frac{1}{4} \sum_{n=1}^{N} \mathrm{SNR}^{1-\alpha_{N}(k-1)+n\left|\tilde{x}_{k, n}\right|^{2}}\right)}\right. \\
& \leq \log _{2}\left(1+\frac{1}{2^{M N}} \sum_{\mathbf{s}^{\prime} \neq \mathbf{s}} e^{-\frac{\left|\min \left\{\tilde{x}_{k}\right\}\right|^{2}}{4} \sum_{n=1}^{N} \mathrm{SNR}^{1-\alpha} \alpha_{N(k-1)+n}}\right) .
\end{aligned}
$$


For large SNR both bounds have the same behavior, and thus we have that

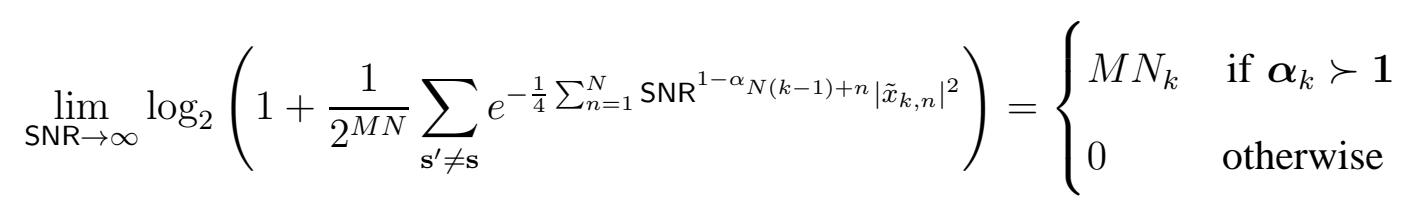

where $\boldsymbol{\alpha}_{k}=\left(\alpha_{N(k-1)+1}, \ldots, \alpha_{N k}\right)^{T}$ and hence

$$
\overline{P_{e}(\mathrm{SNR} \mid \mathbf{H})} \dot{\leq} \exp \left(-B L M \log 2 E_{\delta}(\boldsymbol{\alpha})\right)
$$

where

$$
E_{\delta}(\boldsymbol{\alpha}) \triangleq 1-\frac{R}{M}-\frac{N}{B} \sum_{k=1}^{K} \mathbb{1}\left\{\boldsymbol{\alpha}_{k} \succeq \mathbf{1}-\boldsymbol{\delta}\right\}=1-\frac{R}{M}-\frac{1}{K} \sum_{k=1}^{K} \mathbb{1}\left\{\boldsymbol{\alpha}_{k} \succeq \mathbf{1}-\boldsymbol{\delta}\right\}
$$

and $\boldsymbol{\delta}=(\delta, \ldots, \delta) \in \mathbb{R}_{+}^{N}$. We now define the large SNR error event as

$$
\begin{aligned}
\mathcal{E}_{\delta} & =\left\{\boldsymbol{\alpha} \in \mathbb{R}^{B} \quad: \quad E_{\delta}(\boldsymbol{\alpha}) \leq 0\right\} \\
& =\left\{\boldsymbol{\alpha} \in \mathbb{R}^{B} \quad: \quad \sum_{k=1}^{K} \mathbb{1}\left\{\boldsymbol{\alpha}_{k} \succeq \mathbf{1}-\boldsymbol{\delta}\right\} \geq K\left(1-\frac{R}{M}\right)\right\} .
\end{aligned}
$$

Using the previous results we write that,

$$
\begin{aligned}
\overline{P_{e}(\mathrm{SNR})} & \leq \int_{\boldsymbol{\alpha} \in \mathbb{R}_{+}^{B}} \mathrm{SNR}^{-m \sum_{b=1}^{B} \alpha_{b}} \min \left\{1, \exp \left(-B L M \log 2 E_{\delta}(\boldsymbol{\alpha})\right)\right\} d \boldsymbol{\alpha} \\
& =\int_{\boldsymbol{\alpha} \in \mathcal{E}_{\delta} \cap \mathbb{R}_{+}^{B}} \mathrm{SNR}^{-m \sum_{b=1}^{B} \alpha_{b}} d \boldsymbol{\alpha} \\
& +\int_{\boldsymbol{\alpha} \in \mathcal{E}_{\delta}^{\mathcal{C}} \cap \mathbb{R}_{+}^{B}} \mathrm{SNR}^{-m \sum_{b=1}^{B} \alpha_{b}} \exp \left(-B L M \log 2 E_{\delta}(\boldsymbol{\alpha})\right) d \boldsymbol{\alpha}
\end{aligned}
$$

In a similar way to the proof of Lemma 1 the probability of two randomly chosen codewords over $\mathcal{S}$ being the same is strictly greater than zero, and goes to zero only for $L \rightarrow \infty$. We now study how large $L$ has to be in order for this event not to dominate the overall error probability. If we let

$$
\lambda=\lim _{\mathrm{SNR} \rightarrow \infty} \frac{L}{\log \mathrm{SNR}}
$$

we can write

$$
\begin{aligned}
\overline{P_{e}(\mathrm{SNR})} & =\int_{\boldsymbol{\alpha} \in \mathcal{E}_{\delta} \cap \mathbb{R}_{+}^{B}} \mathrm{SNR}^{-m \sum_{b=1}^{B} \alpha_{b}} d \boldsymbol{\alpha} \\
& +\int_{\boldsymbol{\alpha} \in \mathcal{E}_{\delta}^{c} \cap \mathbb{R}_{+}^{B}} \exp \left(-\log \mathrm{SNR}\left[-m \sum_{b=1}^{B} \alpha_{b}+\lambda B M \log 2 E_{\delta}(\boldsymbol{\alpha})\right]\right) d \boldsymbol{\alpha}
\end{aligned}
$$


Therefore, the overall random coding exponent is given by the minimum of the exponents of (52) and (53),

$$
d_{\mathcal{X}}(R) \geq d_{\mathcal{X}}^{(r)}(R)=\sup _{\delta>0} \min \left\{d_{\mathcal{X}}^{(r), \infty}(R), d_{\mathcal{X}}^{(r), \lambda}(R)\right\}
$$

where

$$
d_{\mathcal{X}}^{(r), \infty}(R)=\inf _{\boldsymbol{\alpha} \in \mathcal{E}_{\delta} \cap \mathbb{R}_{+}^{B}} m\left\{\sum_{b=1}^{B} \alpha_{b}\right\}
$$

is the exponent corresponding to (52) and

$$
d_{\mathcal{X}}^{(r), \lambda}(R)=\inf _{\boldsymbol{\alpha} \in \mathcal{E}_{\delta}^{\mathcal{c}} \cap \mathbb{R}_{+}^{B}}\left\{m \sum_{b=1}^{B} \alpha_{b}+\lambda B M \log 2 E_{\delta}(\boldsymbol{\alpha})\right\}
$$

is the exponent that characterizes the effect of finite length (53). Following similar arguments to the ones used in Lemma 1, it is not difficult to show that the first infimum is achieved by $\kappa$ vectors $\boldsymbol{\alpha}_{k} \succeq \mathbf{1}-\boldsymbol{\delta}$, where $\kappa$ is the unique integer such that

$$
\kappa-1<\left\lceil K\left(1-\frac{R}{M}\right)\right] \leq \kappa
$$

resulting in the exponent being

$$
d_{\mathcal{X}}^{(r), \infty}(R)=(1-\delta) m N\left\lceil\frac{B}{N}\left(1-\frac{R}{M}\right)\right\rceil .
$$

As for the second exponent (56), we can rewrite it as follows

$$
\begin{aligned}
d_{\mathcal{X}}^{(r), \lambda}(R) & =\lambda B M \log 2\left(1-\frac{R}{M}\right) \\
& +\inf _{\boldsymbol{\alpha} \in \mathcal{E}_{\delta}^{\mathcal{C}} \cap \mathbb{R}_{+}^{B}}\left\{m \sum_{b=1}^{B} \alpha_{b}-\lambda B M \log 2 \frac{1}{K} \sum_{k=1}^{K} \mathbb{1}\left\{\boldsymbol{\alpha}_{k} \succeq \mathbf{1}-\boldsymbol{\delta}\right\}\right\} \\
& =\lambda B M \log 2\left(1-\frac{R}{M}\right) \\
& +m \inf _{\boldsymbol{\alpha} \in \mathcal{E}_{\delta}^{\complement} \cap \mathbb{R}_{+}^{B}}\left\{\sum_{k=1}^{K}\left(\sum_{n=1}^{N} \alpha_{k, n}-\frac{\lambda N M \log 2}{m} \mathbb{1}\left\{\boldsymbol{\alpha}_{k} \succeq \mathbf{1}-\boldsymbol{\delta}\right\}\right)\right\}
\end{aligned}
$$

The constraint set $\mathcal{E}_{\delta}^{c}$ is defined as follows

$$
\mathcal{E}_{\delta}^{c}=\left\{\boldsymbol{\alpha} \in \mathbb{R}^{B} \quad: \quad \sum_{k=1}^{K} \mathbb{1}\left\{\boldsymbol{\alpha}_{k} \succeq \mathbf{1}-\boldsymbol{\delta}\right\}<K\left(1-\frac{R}{M}\right)\right\} .
$$

We distinguish two cases. When $0 \leq \lambda N M \log 2<m$ then the terms

$$
\sum_{n=1}^{N} \alpha_{k, n}-\frac{\lambda N M \log 2}{m} \mathbb{1}\left\{\boldsymbol{\alpha}_{k} \succeq \mathbf{1}-\boldsymbol{\delta}\right\}
$$


attain its minimum value for $\boldsymbol{\alpha}_{k}=\mathbf{0}$. On the other hand, when $\lambda N M \log 2 \geq m$, the constraint set dictates that there should be

$$
\kappa=\left\lfloor K\left(1-\frac{R}{M}\right)\right\rfloor
$$

vectors $\boldsymbol{\alpha}_{k} \succeq \mathbf{1}-\boldsymbol{\delta}$, and the infimum becomes

$$
\lambda B M \log 2\left(1-\frac{R}{M}\right)+m\left\lfloor K\left(1-\frac{R}{M}\right)\right\rfloor\left(N(1-\delta)-\frac{\lambda N M \log 2}{m}\right) .
$$

Combining the previous results and noting that the supremum in (54) is achieved for $\delta \rightarrow 0$, we find the desired result. 


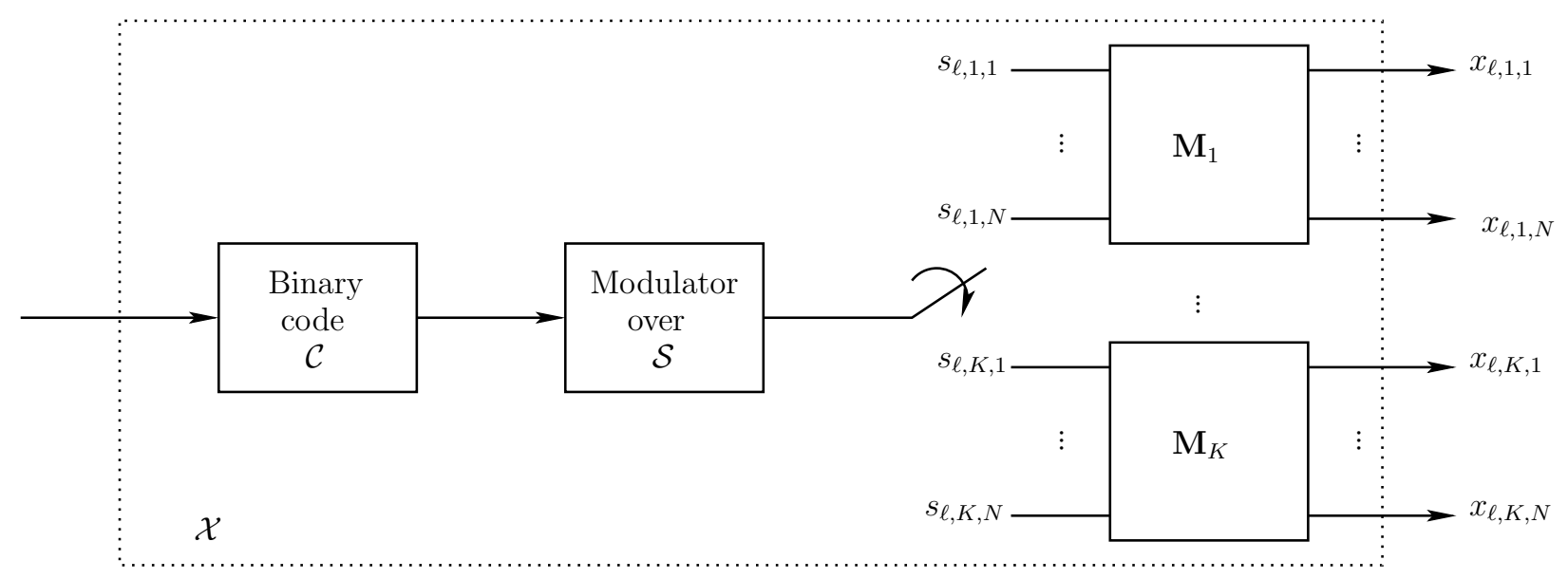

Fig. 1. Block diagram for coded modulation with $K$ rotated constellations with rotation matrices $\mathbf{M}_{1}, \ldots, \mathbf{M}_{K}$. 


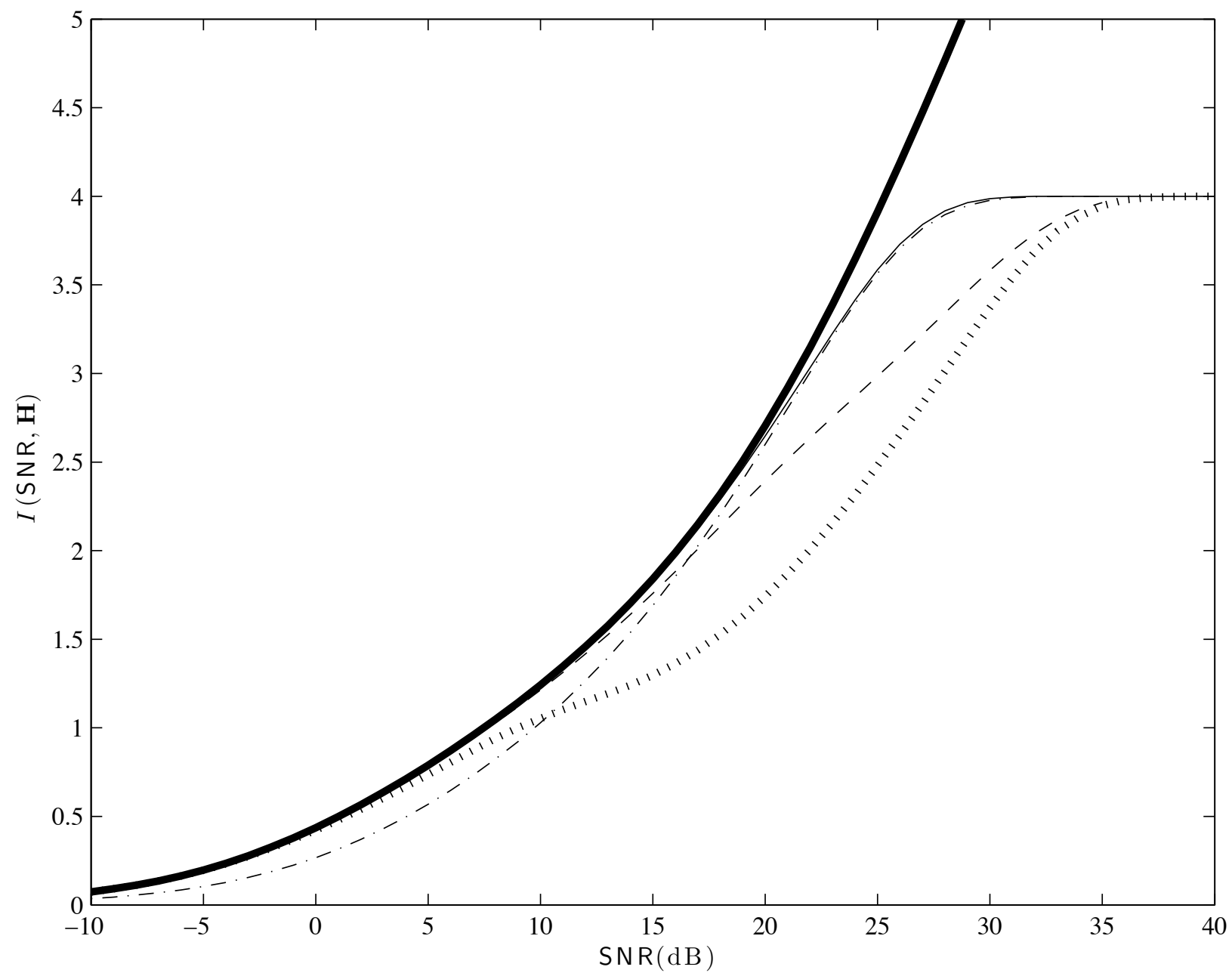

Fig. 2. Instantaneous mutual information $I(\mathrm{SNR}, \mathrm{H})$ (bits/channel use) in a block-fading channel with $B=4$ blocks and $h_{1}=1.5$ and $h_{2}=h_{3}=h_{4}=0.1$ with Gaussian inputs (thick solid) and rotated 16-QAM inputs with the optimal Krüskemper (thin solid), mixed (thin dash-dotted), 2 independent 2-dimensional cyclotomic rotations (thin dashed) and no rotations (thick dotted). 


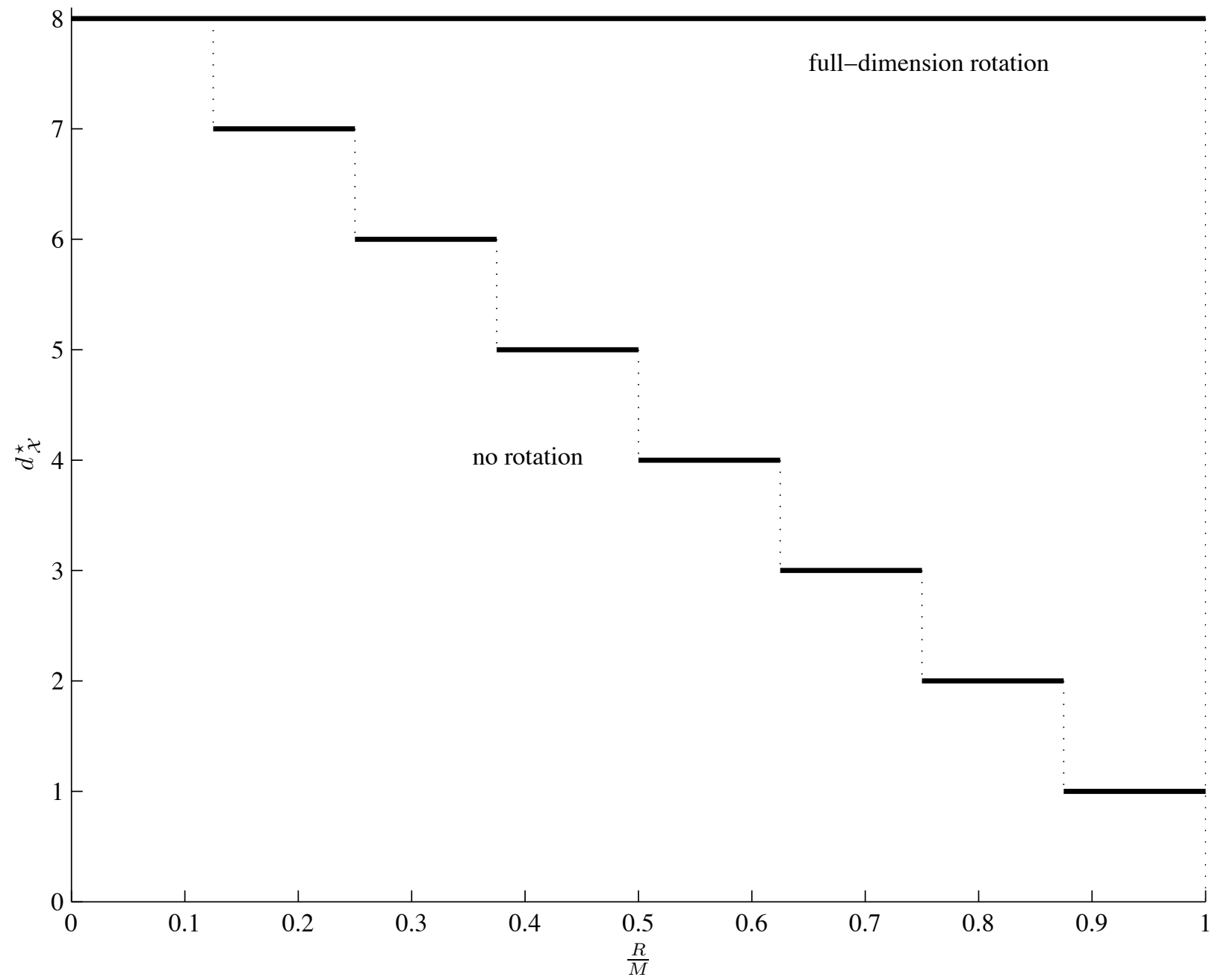

Fig. 3. Diversity reliability exponents for $B=8$ and $m=1$. Optimal exponent (13) and Singleton bound (14). 


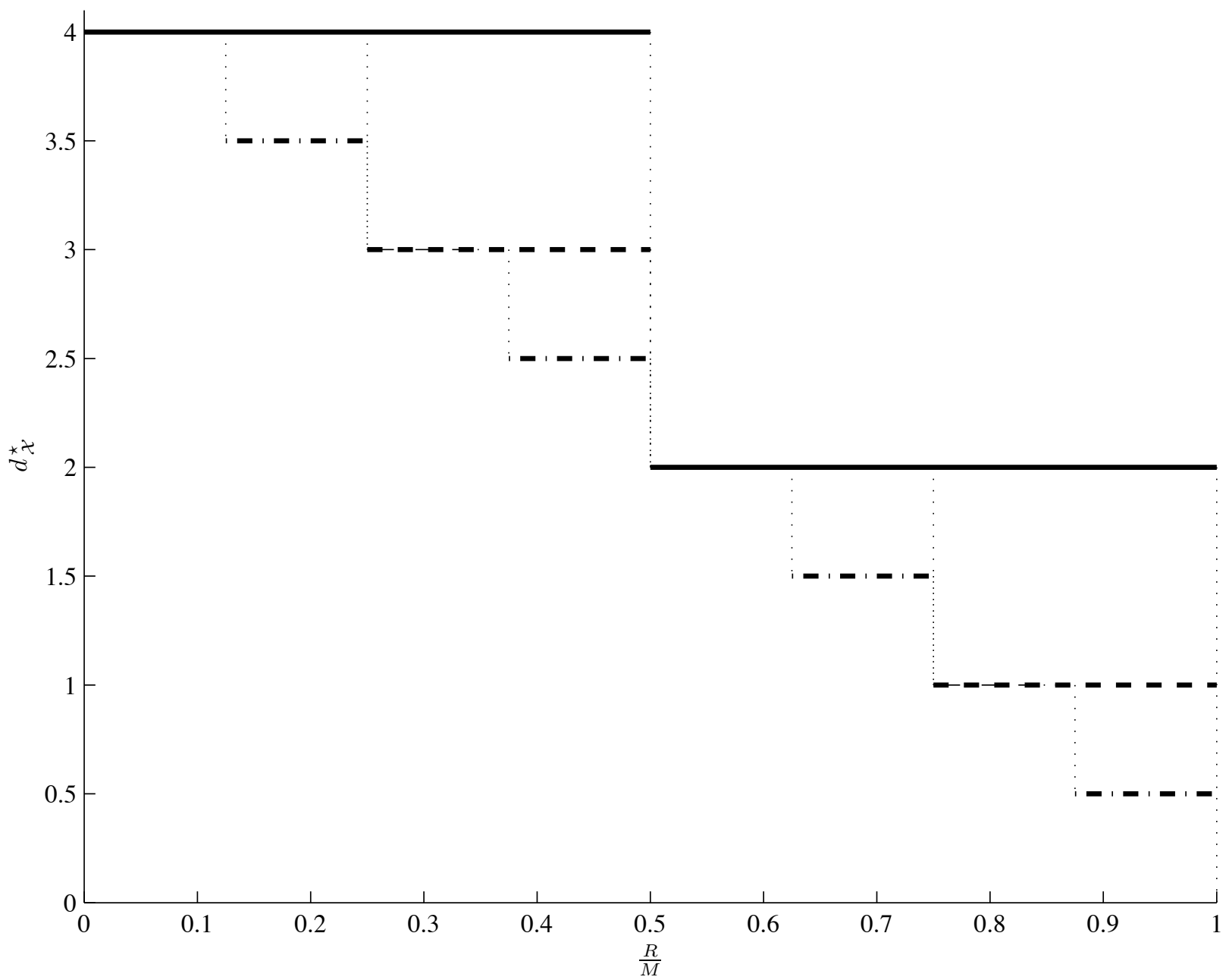

Fig. 4. Reliability exponents for $B=8, m=0.5$ and rotations of dimensions $N=1$ (dash-dotted), $N=2$ (dashed) and $N=4$ (solid). 


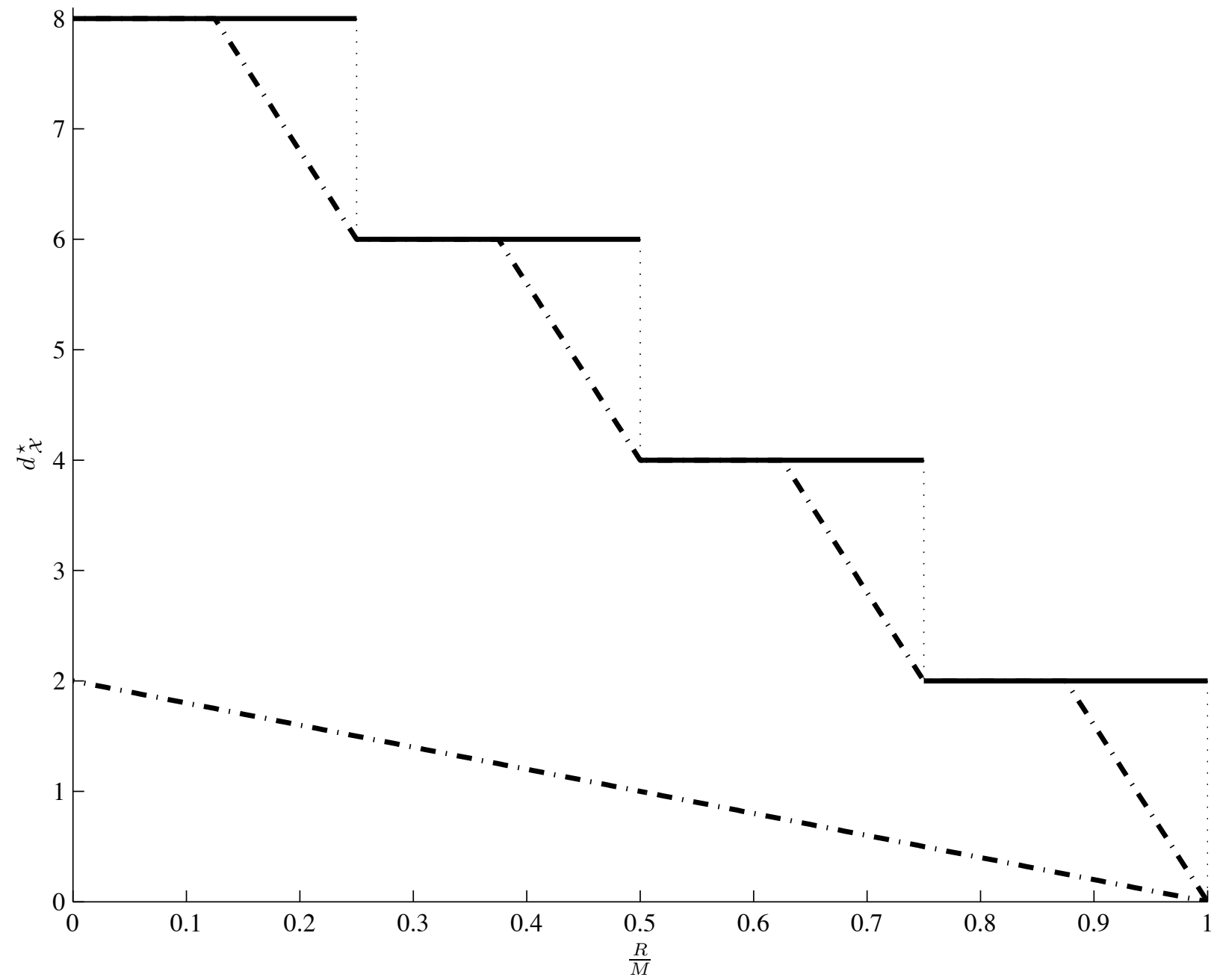

Fig. 5. Reliability exponents for $B=8, m=1$ and rotations of dimensions $N=2$. The random coding exponents for $\lambda M \log 2=\frac{m}{2 N}$ (lower dash-dotted curve) and $\lambda M \log 2=\frac{4 m}{N}$ (upper dash-dotted curve) are also shown. 


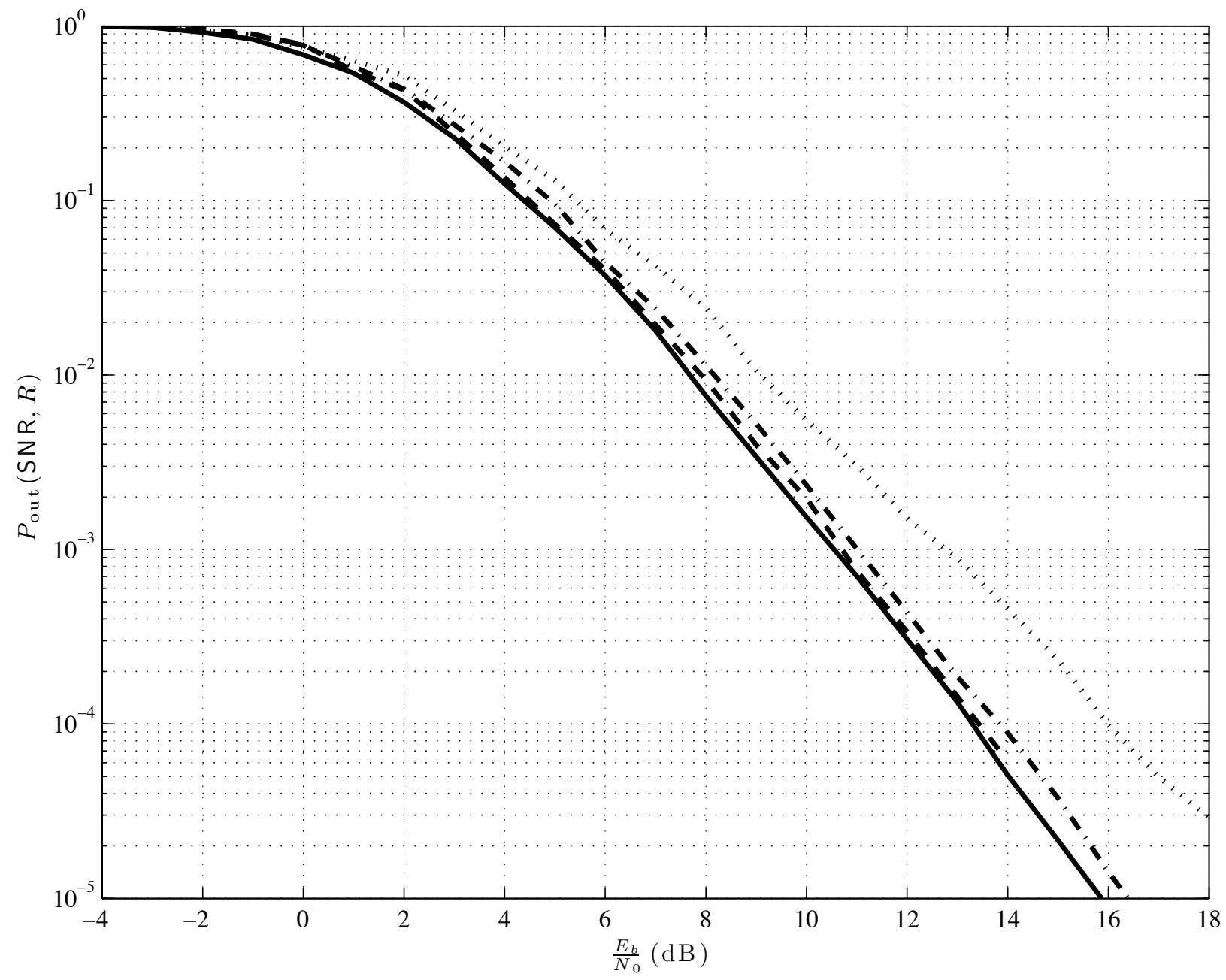

Fig. 6. Outage probability for $R=1$ bits per channel use in a block-fading channel with $B=4, m=1$, with Gaussian (solid line), rotated QPSK inputs with one Krüskemper rotation of dimension $N=4$ (dashed line), rotated QPSK inputs with two cyclotomic rotations of dimension $N=2$ (dash-dotted) and unrotated QPSK inputs (dotted). 


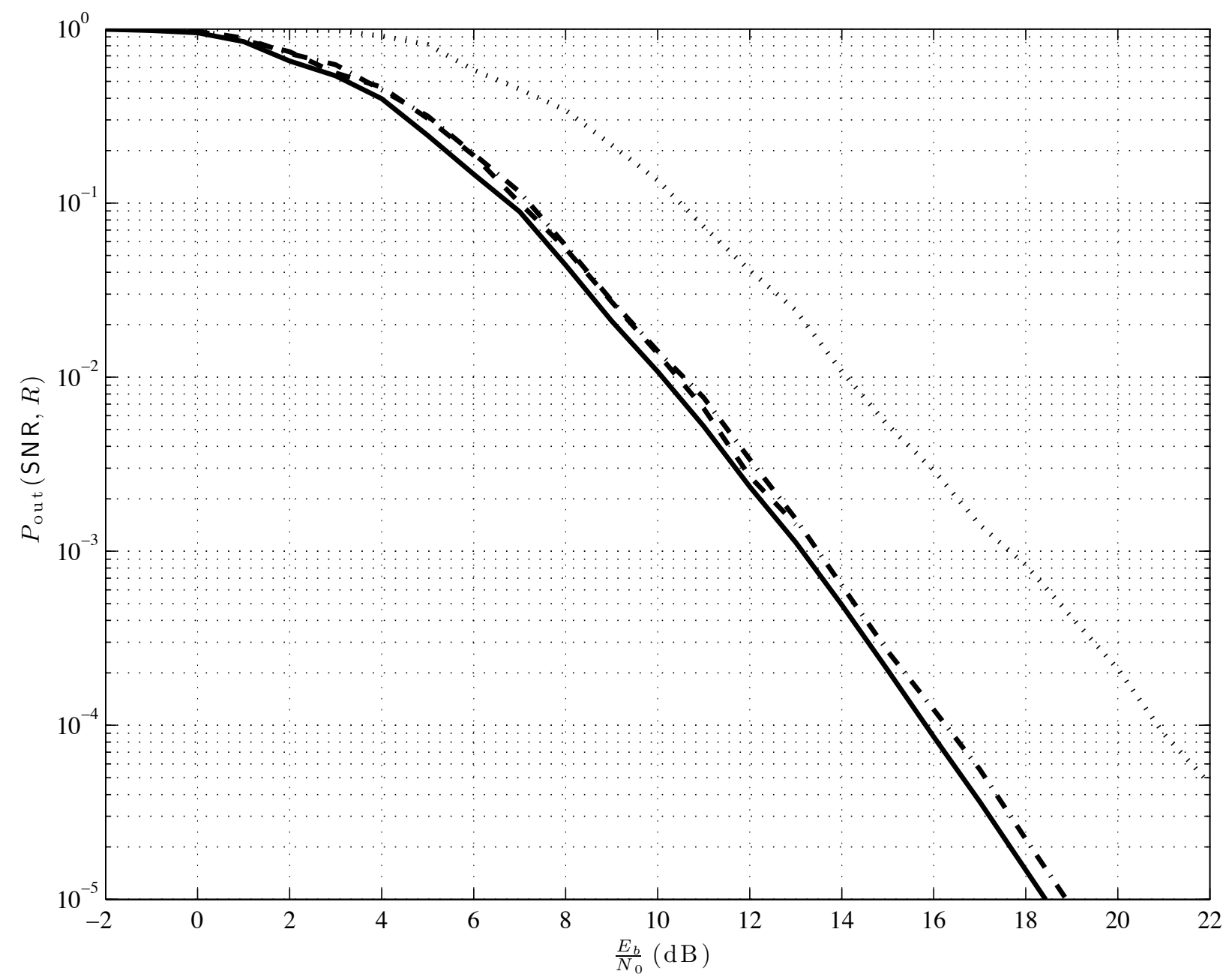

Fig. 7. Outage probability for $R=2$ bits per channel use in a block-fading channel with $B=4, m=1$, with Gaussian(solid line), rotated 16-QAM inputs with one Krüskemper rotation of dimension $N=4$ (dashed line), rotated 16-QAM inputs with two cyclotomic rotations of dimension $N=2$ (dash-dotted) and unrotated 16-QAM inputs (dotted). 


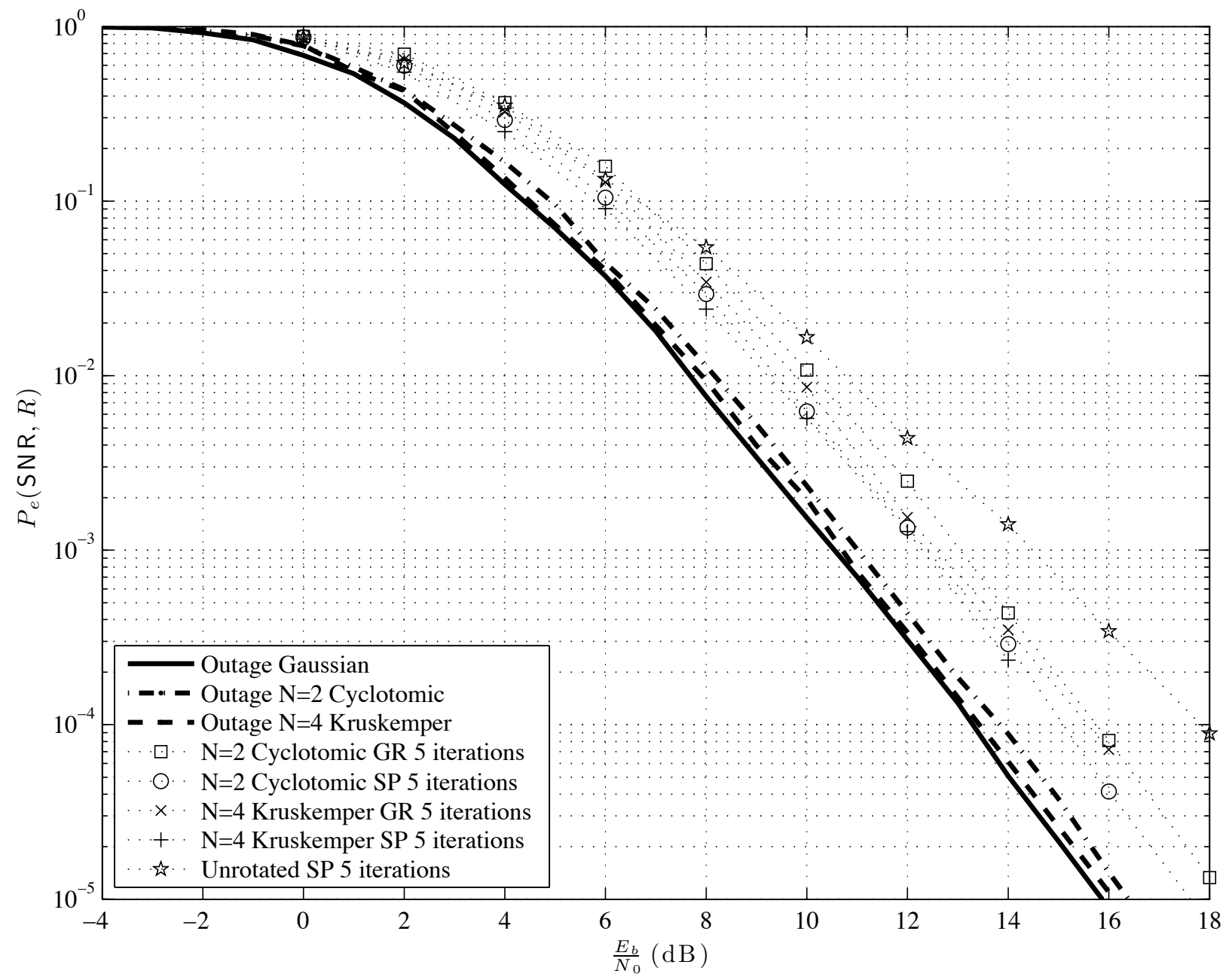

Fig. 8. Error probability for $R=1$ bits per channel use in a block-fading channel with $B=4, m=1$ using the $(5,7)_{8}$ convolutional code and QPSK modulation with Gray (GR) and set-partitioning (SP) labeling. The outage probabilities with Gaussian inputs (thick solid line), rotated QPSK inputs with one Krüskemper rotation of dimension $N=4$ (dashed line), rotated QPSK inputs with two cyclotomic rotations of dimension $N=2$ (dash-dotted) are shown for reference. 


\section{REFERENCES}

[1] G. Battail, "Rotating a redundant constellation in signal space against channel fluctuations," Int. Conf. Commun. Tech., Beijing, China, Jul.. Also ENST, Paris, France, rapport interne, 1989 (postponed until 1990).

[2] K. Boulle and J.-C. Belfiore, "Modulation schemes designed for the rayleigh channel," in Proc. Conf. Inf. Sciences and Systems, Princeton University, Princeton, NJ, Mar. 1992, pp. 288-293.

[3] J. Boutros and E. Viterbo, "Signal space diversity: a power-and bandwidth-efficient diversitytechnique for the Rayleigh fading channel," IEEE Trans. Inf. Theory, vol. 44, no. 4, pp. 1453-1467, Jul. 1998.

[4] E. Bayer-Fluckiger, F. Oggier, E. Viterbo, "New algebraic constructions of rotated $\mathbb{Z}^{n}$-lattice constellations for the rayleigh fading channel,” IEEE Trans. on Inf.. Theory, vol. 50, no. 4, pp. 702-714, Apr. 2004.

[5] F. Oggier and E. Viterbo, "Algebraic number theory and code design for rayleigh fading channels," Foundations and Trends in Communications and Information Theory, vol. 1, pp. 333-415, 2004.

[6] F. Oggier, Algebraic methods for channel coding, Ph.D. thesis, Ecole Polytechnique Fédérale de Lausanne, 2005.

[7] N. Gresset, J. Boutros and L. Brunel, "Optimal linear precoding for BICM over MIMO channels," in Proc. 2004 IEEE Int. Symp. Inf. Theory, Chicago, IL, Jun,-Jul. 2004.

[8] G. M. Kraidy, N. Gresset, and J. J. Boutros, "Information theoretical versus algebraic constructions of linear unitary precoders for non-ergodic multiple antenna channels," Canadian Workshop Inf. Theory, Montreal, Jun., 2005.

[9] L. H. Ozarow, S. Shamai and A. D. Wyner, "Information theoretic considerations for cellular mobile radio," IEEE Trans. on Vehicular Tech., vol. 43, no. 2, pp. 359-378, May 1994.

[10] E. Viterbo and J. Boutros, "A universal lattice decoder for fading channels," IEEE Trans. Inf. Theory, vol. 45, no. 4, pp. 1639-1642, Jul. 1999.

[11] J. Boutros, N. Gresset, L. Brunel and M. Fossorier, "Soft-input soft-output lattice sphere decoder for linear channels," IEEE Global Commun. Conf., Dec. 2003.

[12] J. Proakis, Digital Communications, McGraw-Hill, 1995.

[13] M. Abramowitz and I. A. Stegun, Handbook of Mathematical Functions with Formulas, Graphs and Mathematical Tables, New York: Dover Press, 1972.

[14] M. K. Simon and M. S. Alouini, Digital Communication over Fading Channels, John Wiley: New York, 2000.

[15] S. Verdú and T. S. Han, “A general formula for channel capacity,” IEEE Trans. Inf. Theory, vol. 40, no. 4, pp. 1147-1157, Jul. 1994.

[16] E. Biglieri, J. Proakis and S. Shamai, "Fading channels: information-theoretic and communications aspects," IEEE Trans. Inf. Theory, vol. 44, no. 6, pp. 2619 -2692, Oct. 1998.

[17] T. M. Cover and J. A. Thomas, Elements of Information Theory, Wiley Series in Telecommunications, 2nd edition, 2006.

[18] E. Viterbo and F. Oggier, "Tables of algebraic rotations," http://www.tlc.polito.it/ viterbo

[19] J. H. Conway and N. J. A. Sloane, Sphere packings, lattices and groups, Springer, 3rd edition, 1999.

[20] A. Guillén i Fàbregas and G. Caire, "Coded modulation in the block-fading channel: Coding theorems and code construction,” IEEE Trans. Inf. Theory, vol. 52, no. 1, pp. 262-271, Jan. 2006.

[21] K. D. Nguyen, A. Guillén i Fàbregas and L. K. Rasmussen, "A Tight Lower Bound to the Outage Probability of BlockFading Channels," submitted to IEEE Trans. Inf. Theory, 2007.

[22] G. Caire, G. Taricco, and E. Biglieri, "Bit-interleaved coded modulation," IEEE Trans. Inf. Theory, vol. 44, no. 3, pp. 927-946, May 1998.

[23] J.J. Boutros, E. Calvanese Strinati, and A. Guillén i Fàbregas, "Turbo code design for block fading channels," 42nd Allerton Conf. Commun., Control and Computing, Monticello, IL, Sept.-Oct. 2004.

[24] L. Zheng and D. Tse, "Diversity and multiplexing: A fundamental tradeoff in multiple antenna channels," IEEE Trans. Inf. Theory, vol. 49, no. 5, May 2003.

[25] A. Dembo and O. Zeitouni, Large Deviations Techniques and Applications, Number 38 in Applications of Mathematics. Springer Verlag, 2nd edition, Apr. 1998. 